(2) that the simple form of dispersion formula (without the Lorentz-Hartree correction) is correct for transmission in an unpolarised medium like the ionosphere.

This is the conclusion arrived at by Darwin' ${ }^{2}$ on theoretical grounds.

T. L. ECKersLey.

Research Department,

Marconi's Wireless Telegraph Co., Ltd., Chelmsford.

Dec. 19.

${ }^{2}$ Barkhausen, Phys. Z., 20, 401-403 ; 1919. Proc. I.R.E., 18, No. 7, July 1930. Shellang, Bell Tel. J., Aug. 1930. Eckersley, Phil. Mag. 49,$1250 ; 1925$. NATURE, 122, 768; 1928. Burton, NATURE, 126, 55 1930. Barton and Boardman, Bell. Tel. J., 12, 498-516; 1933.

Darwin, NATURE, 133, 62; 1934 .

\section{Over-Potential of the Hydrogen Isotopes}

Autнобян the separation of the two hydrogen isotopes which occurs on electrolysis must be closely connected with their over-potentials, no measurement of these has been published. A comparison of the deuterium over-potential with that of hydrogen can be of value in elucidating the general mechanism of hydrogen over-potential itself and, as part of an experimental investigation of this, measurements have been made on deuterium. In order to eliminate errors due to the resistance of the electrolyte and to violent gas evolution, it is essential that very low eurrent densities should be used, and the electrolyte must be free from traces of oxygen or dissolved impurities. The over-potential measurements were made under conditions similar to those described in earlier papers ${ }^{1}$, but it was a matter of some experimental difficulty to realise these with the small quantities of electrolyte available (1 c.c.). The results obtained with a mercury cathode in a $0.2 \mathrm{~N}$ sulphuric acid electrolyte are shown in Fig. 1.

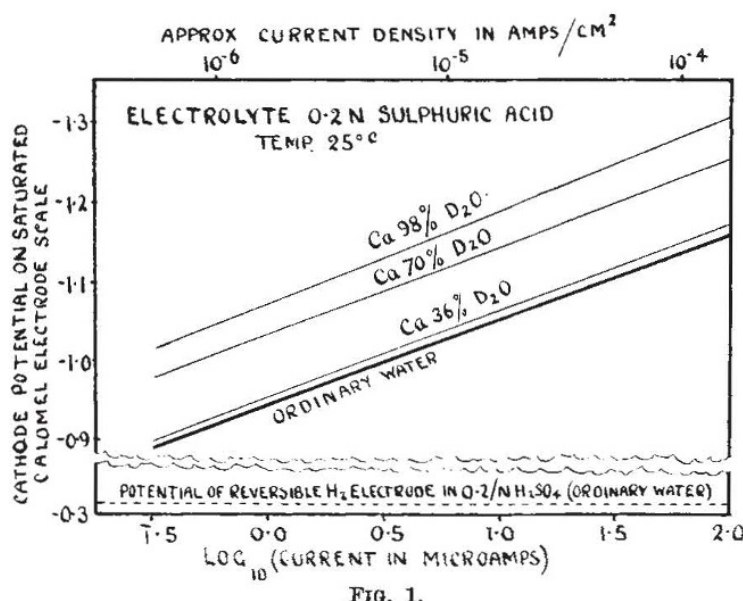

Fig. 1.

It is apparent that both isotopes give the same linear relation between current density and overpotential, and that the slope of the line is nearly the same in each case cas $^{2} \alpha=-2 \cdot 3 \mathrm{RT} d \log i / d V=$ about $0 \cdot 5$ ). The irreversible potential of the deuterium is, however, considerably higher than that of hydrogen (c. 0.13 volts more negative). For 100 per cent heavy water the difference would be slightly greater. (The over-potential is, strictly, the potential difference between an irreversible electrode and a reversible electrode working in the same electrolyte. Measure. ments made in this laboratory by Dr. K. E. Grew show, however, that the potential difference between a reversible deuterium electrode and a reversible hydrogen electrode is small.)

From these measurements it is clear that the over-potential difference between the isotopes is sufficiently great to explain the separation which occurs on electrolysis. From the curves we may calculate a possible separation factor under these optimum conditions. For example, at a cathode potential of -1.05 volts sat. cal.

the rate of evolution of $\mathrm{H}_{2}=\frac{9.23 \times 10^{-8} \mathrm{amp} .}{0.668 \times 10^{-6} \mathrm{amp} .}=\frac{13.8}{\mathrm{l}}$.

We have also measured the temperature coefficient of the deuterium over-potential and find that it is greater than for hydrogen, showing that the efficiency of separation will decrease as the temperature increases.

As would be expected, the actual separation factor observed during the preparation of heavy water is considerably less than the possible one suggested above. (It varies from c. 3 at a reversible electrode to $c .8^{3,4}$.) Under the usual conditions of electrolysis, there are many factors such as high current density, high local temperature, concentration polarisation, low over-potential properties of the electrode surface, interchange, etc., all of which tend to lower the efficiency. The bearing of these results on the mechanism of over-potential will be discussed elsewhere.

It is with much pleasure that we express our thanks to Drs. A. and L. Farkas for analysing the heavy water and to Imperial Chemical Industries, Ltd., for a grant.

Laboratory of Physical Chemistry,

F. P. Bowden.

H. F. KENYON. Cambridge.

Nov. 27.

${ }^{1}$ Bowden, Proc. Roy. Soc., A, 125, 446 ; 1929.

Bowden, Proc. Roy. Soc., A, 126, $108 ; 1929$.

Tarkas and Farkas, Proc. Roy. Soc. A 146, 623; 1934

4 Eyring and Topley J. Chem Phys., 2, 217; 1934. Bell and Wolfenden, Nature, 133, 25; 1934.

\section{Fundamental Dimensions of $\mu_{0}$ and $K_{0}$ in} Electrical Science

THE recent work of committees both national and international upon the fundamental units and definitions in electrical science has shown that a considerable further step in advance, by removing difficulties, would result from the discovery of the dimensions of $\mu_{0}$ and $K_{0}$, the magnetic permeability and electric permittivity or specific inductive capacity of the medium. Some writers have recommended that the dimensions of one of these should be chosen arbitrarily in order to get this simplification, the result of which would be that the difference in physical dimensions of the various quantities, when measured in electrostatic and electromagnetic units, would disappear. Difference in size of unit causes no confusion, but the difference in dimensions due to changing the scale of measurement has been a great cause of confusion.

Electrical science is based fundamentally upon the definitions of quantity of electricity $e$, magnetic pole $m$ and electric current $i$. The definitions of these three quantities are given by the three equations:

$$
\begin{aligned}
& \text { force }=e e^{\prime} / K_{0} r^{2} \ldots \text { (1) force }=m m^{\prime} / \mu_{0} r^{2} . \\
& \quad \text { and } i=d e / d t . .(3) \text {. }
\end{aligned}
$$

